\title{
Prevalence of allergic rhinitis and its impact on the use of emergency care services in a group of children and adolescents with moderate to severe persistent asthma
}

\author{
Laura M. L. B. F. Lasmar, ${ }^{1}$ Paulo A. M. Camargos, ${ }^{2}$ Alexandre Beraldo Ordones, ${ }^{3}$ \\ Guilherme Rache Gaspar, ${ }^{4}$ Eduardo Goulart Campos, ${ }^{5}$ Gustavo Augusto Ribeiro ${ }^{6}$
}

\begin{abstract}
Objective: To assess the prevalence of allergic rhinitis and the factors associated with the use of emergency care services by children and adolescents with acute asthma submitted to inhaled corticosteroid therapy.

Methods: A cross-sectional study was conducted with 126 patients treated with beclomethasone dipropionate for 3 years. The factors associated with emergency care services in the third year of beclomethasone dipropionate treatment were assessed using logistic regression models.

Results: The prevalence of allergic rhinitis amounted to $74.6 \%$ (95\%CI 65.9-81.7). The presence of allergic rhinitis $(\mathrm{OR}=2.98,95 \% \mathrm{CI} 1.10-8.06)$ and asthma severity $(\mathrm{OR}=2.09,95 \% \mathrm{CI} 1.05-4.44)$ were independent factors for emergency care services.

Conclusion: The prevalence of allergic rhinitis was high and that, combined with asthma severity, constituted the major risk factor for the necessity of emergency care services. Health professionals should attempt to make an early diagnosis of allergic rhinitis in asthmatic patients.
\end{abstract}

J Pediatr (Rio J). 2007;83(6):555-561: Asthma, allergic rhinitis, emergency care services, inhaled corticosteroids.

\section{Introduction}

Asthma and allergic rhinitis (AR) are manifestations of united airway disease. ${ }^{1,2}$ The medical literature has shown that $\mathrm{AR}$ is found in 75 to $80 \%$ of asthmatic patients. ${ }^{3,4}$

Emergency care services account for only $1 \%$ of direct costs related to $A R$, but for $62 \%$ of expenses on asthma management. ${ }^{5,6}$ Clinical observation and literature data demonstrate that an adequate approach to upper airway inflammation is paramount to the successful management of asthmatic patients. ${ }^{1,2}$ It is common knowledge that the association between asthma and AR results in a larger prescription of asthma drugs, higher costs, and wider use of health services. ${ }^{7,8}$ Moreover, the need of emergency care services due to exacerbations of asthma indicates poor management of asthma among patients treated with prophylactic drugs. ${ }^{9}$

In our review of the literature, we did not find any Brazilian studies on the impact of AR on emergency care services in asthmatic patients treated with inhaled corticosteroids.

The aim of the present study is to assess the prevalence of $A R$ in asthmatic patients treated at a referral outpatient

1. Doutora. Professora adjunta. Departamento de Pediatria, Faculdade de Medicina de Minas Gerais, Belo Horizonte, MG, Brazil.

2. Professor titular, Departamento de Pediatria, Faculdade de Medicina de Minas Gerais, Belo Horizonte, MG, Brazil.

3. Acadêmico de Medicina, Universidade Federal de Minas Gerais (UFMG), Belo Horizonte, MG, Brazil. Bolsista, Iniciação Científica, Conselho Nacional de Desenvolvimento Científico e Tecnológico (CNPq, 105680/2005-2).

4. Acadêmico de Medicina, UFMG, Belo Horizonte, MG, Brazil. Bolsista, Iniciação Científica, Fundação de Amparo à Pesquisa do Estado de Minas Gerais (FAPEMIG).

5. Médico, UFMG, Belo Horizonte, MG, Brazil. Bolsista, Iniciação Cientifica, FAPEMIG.

6. Médico, UFMG, Belo Horizonte, MG, Brazil. Bolsista, Iniciação Científica, CNPq.

No conflicts of interest declared concerning the publication of this article.

Suggested citation: Lasmar LM, Camargos PA, Ordones AB, Gaspar GR, Campos EG, Ribeiro GA. Prevalence of allergic rhinitis and its impact on the use of emergency care services in a group of children and adolescents with moderate to severe persistent asthma. J Pediatr (Rio J). 2007;83(6):555-561.

Manuscript received July 11 2007, accepted for publication Oct 032007.

doi $10.2223 / J P E D .1727$ 
clinic and to determine the factors associated with the use of emergency care services in a group of children and adolescents put on prophylactic treatment with beclomethasone dipropionate.

\section{Methods}

A cross-sectional study was conducted with 126 patients treated at Campos Sales Secondary Referral Unit of Pediatric Pulmonology, affiliated with the Municipal Department of Health of Belo Horizonte, Brazil. This unit piloted the asthma control program in Belo Horizonte - Wheezing Child Program -, using beclomethasone as the drug of choice since 1994, when a standard protocol was adopted for the follow-up of asthmatic patients.

The data were collected in 2005 by retrospective analysis of the medical records of all patients admitted to this outpatient clinic between 1994 and 2005. The data on those patients who met the inclusion criteria were transferred to a customized protocol.

Patients aged 3 to 17 years, with moderate to severe persistent asthma, followed up for 3 years in a row, were included in the study. Patients with other underlying diseases were excluded.

The diagnosis and classification of asthma severity were written down in the medical records and were based on the criteria established by the Global Initiative for Asthma (GINA). ${ }^{9}$ The diagnosis and classification of AR were based on clinical criteria proposed by experts from several countries in partnership with the World Health Organization, known as Allergic Rhinitis and Its Impact on Asthma (ARIA). ${ }^{10}$ The symptoms of $A R$ included the single or combined presence of itchy nose and/or itchy oropharynx, serous or seromucous rhinorrhea, sneezing, itchy eyes, and nasal congestion. AR was classified as persistent when these signs and symptoms lasted for 4 or more days a week and for 4 weeks. ${ }^{10}$

Emergency care services and asthma exacerbations which are telltale signs of impact in the Wheezing Child Program - were validated by counter-referral forms and recorded on the protocol for emergency care of asthmatic children. Exacerbations were defined according to the protocol of this program, which is based on GINA criteria. ${ }^{9,11}$

Patients with exacerbations were treated at emergency care units (ECU) of Belo Horizonte. With the implementation of the Wheezing Child Program, the ECU team obtained qualification in the management of acute asthma attacks, and all procedures were standardized by the protocol. ${ }^{11}$

After emergency care at the ECU, patients were sent back to the referral unit, and a counter-referral form with all services received and all drugs prescribed was provided. ${ }^{11}$

At Campos Sales outpatient clinic, a personalized action plan, which includes an educational approach, is used as one of the treatment mainstays, and patients receive an ID card with all detailed information about the prophylactic treatment and action plan. ${ }^{11}$

\section{Procedures}

All children who required inhaled corticosteroids received the medication from the outpatient dispensary. Beclomethasone dipropionate is the inhaled corticosteroid used by the asthma control program of Belo Horizonte, and is given out in flasks containing 200 doses, each jet with $250 \mu \mathrm{g}$. Valved spacers (Flumax ${ }^{\circledR}$, Flumax Equipamentos Médicos Ltda., Belo Horizonte, Brazil) were used for administration of the drug, being fitted to the face mask or mouthpiece according to patient's age. Both the drug and the spacer were provided free of charge to the patients. ${ }^{11}$

The pharmacological records of the Wheezing Child Program contains identification information, drug name, amount of flasks, dosage, administration dates, and date of return for a new inhaler. Patients were only provided with a new flask after they returned the first one empty. None of the patients could be registered at two dispensaries. ${ }^{11}$

The rates of beclomethasone compliance were obtained by the following two methods and formulas:

1) percentage rate according to the pharmacological records, calculated by the following formula: number of prescribed doses/number of doses that should be used between the date of dispensation and the date of return $\mathrm{x}$ $100 .^{8}$

2) rate in $\mu \mathrm{g} /$ day: number of $\mu \mathrm{g}$ shown on the flask/number of days between the date of dispensation and the date of return.

The program dispenses dextrochlorpheniramine for the treatment of AR.

\section{Statistical analysis}

First of all, independent variables and their association with the dependent variable (emergency care services in the third year of treatment) were assessed by the odds ratio, using a $95 \%$ confidence interval.

Thereafter, all the variables with a $p \leq 0.20$ in the univariate analysis were added to the logistic regression model. The preliminary model included age range, classification of asthma severity, presence or absence of $A R$ and number of exacerbations.

The exclusion of each variable was determined by Wald's test, and the models were compared using the likelihood ratio. The final model was adjusted by Hosmer-Lemeshow statistic.

The significance level was set as $p<0.05$.

\section{Ethical aspects}

The study protocol was approved by the Research Ethics Committee of Universidade Federal de Minas Gerais, Brazil. 


\section{Results}

In 2005,861 patients were submitted to beclomethasone dipropionate therapy: 576 for less than 3 years and 285 for $\geq$ 3 years, which means that only 126 patients were eligible.

The general characteristics of the study population are shown in Table 1.

Most children were male and their median age was 99.2 months. AR was observed in $74.6 \%$ of the children. None of the patients was referred by basic health services to the outpatient clinic with a prior diagnosis of AR.

Table 2 shows the distribution of patients according to the presence or absence of AR.

Note that the patients did not show statistically significant differences in terms of gender, age range, asthma severity and duration of disease. However, patients with AR had higher beclomethasone compliance rates than those without AR (Table 3).
Note that 44 patients had exacerbations that required emergency care. The presence of AR virtually tripled the necessity for emergency care.

Table 4 shows the final model for emergency care services in the third year of inhaled corticosteroid therapy.

Te presence of AR and asthma severity was regarded as independent factor for the necessity of emergency care. The number of exacerbations lost its statistical significance when adjusted for asthma severity, whereas age range lost its significance when adjusted for AR. The Hosmer-Lemeshow statistic yielded a value of 0.964 , showing that the model was well adjusted. There was no interaction between the analyzed variables.

\section{Discussion}

The present study revealed that $74.6 \%$ of asthmatic children and adolescents developed concomitant AR, the presence of which, combined with asthma severity, was correlated with the necessity of emergency care.

Table 1 - Descriptive characteristics of the study group

\begin{tabular}{|c|c|c|c|c|}
\hline Factors & $\mathbf{n}$ & $\%$ & $95 \% \mathrm{CI}$ & Median (range) \\
\hline Age range (months) & & & & $99.2(40.4-201)$ \\
\hline Maternal education (years of schooling) & & & & $5.0(0-14)$ \\
\hline Income (minimum wages/1994) & & & & $2.0(0-10)$ \\
\hline Age at onset of asthma symptoms (months) & & & & $12(3-132)$ \\
\hline Duration of asthma (months) & & & & $45(0-240)$ \\
\hline \multicolumn{5}{|l|}{ Gender } \\
\hline Male & 79 & 62.7 & & \\
\hline Female & 47 & 37.3 & & \\
\hline \multicolumn{5}{|l|}{ Asthma exacerbations } \\
\hline Present & 44 & 34.9 & $26.8-43.9$ & \\
\hline Absent & 82 & 65.1 & & \\
\hline \multicolumn{5}{|l|}{ Asthma severity } \\
\hline Severe persistent asthma & 55 & 43.7 & $34.2-51.9$ & \\
\hline Moderate persistent asthma & 71 & 53.3 & & \\
\hline \multicolumn{5}{|l|}{ Allergic rhinitis } \\
\hline Present & 94 & 74.6 & $65.9-81.7$ & \\
\hline Absent & 32 & 25.3 & & \\
\hline \multicolumn{5}{|l|}{ Inhaled corticosteroid therapy } \\
\hline Yes & 126 & 100 & & \\
\hline No & 0 & 0 & & \\
\hline \multicolumn{5}{|l|}{ Prior diagnosis of allergic rhinitis } \\
\hline Yes & 0 & 0 & & \\
\hline No & 126 & 0 & & \\
\hline
\end{tabular}

$95 \% \mathrm{CI}=95 \%$ confidence interval. 
558

Table 2 - Distribution of patients according to the presence or absence of allergic rhinitis

\begin{tabular}{|c|c|c|c|}
\hline \multirow[b]{2}{*}{ Variable } & \multicolumn{2}{|c|}{ Allergic rhinitis } & \multirow[b]{2}{*}{$\mathbf{p}$} \\
\hline & Present, n (\%) & Absent, n (\%) & \\
\hline \multicolumn{4}{|l|}{ Gender } \\
\hline Male & $58(61.7)$ & $21(65.6)$ & 0.692 \\
\hline Female & $36(38.3)$ & $11(34.4)$ & \\
\hline \multicolumn{4}{|l|}{ Age range (months) } \\
\hline $40.4-72$ & $23(24.5)$ & $9(28.2)$ & 0.684 \\
\hline$>72$ & $71(75.5)$ & $23(71.8)$ & \\
\hline \multicolumn{4}{|l|}{ Asthma severity } \\
\hline Severe persistent asthma & $44(46.3)$ & $11(35.5)$ & 0.314 \\
\hline \multirow[t]{2}{*}{ Moderate persistent asthma } & $51(53.7)$ & $20(64.5)$ & \\
\hline & Mean \pm SD & Mean \pm SD & \\
\hline Duration of disease & $52.6 \pm 41.4$ & $47.0 \pm 39.3$ & 0.469 \\
\hline $\begin{array}{l}\text { Rate of beclomethasone } \\
\text { compliance in } \%\end{array}$ & $64.42 \pm 27.78$ & $47.41 \pm 31.81$ & 0.007 \\
\hline $\begin{array}{l}\text { Rate of beclomethasone } \\
\text { compliance in } \mu \mathrm{g} / \text { day }\end{array}$ & $452.3 \pm 183.1$ & $311 \pm 218$ & 0.001 \\
\hline
\end{tabular}

$\mathrm{SD}=$ standard deviation.

Table 3 - Factors associated with emergency care services (univariate analysis)

\begin{tabular}{|c|c|c|c|c|}
\hline \multirow[b]{2}{*}{ Factors } & \multicolumn{2}{|c|}{ Emergency care service } & \multirow[b]{2}{*}{$\mathbf{p}$} & \multirow[b]{2}{*}{ OR (95\%CI) } \\
\hline & Yes, n (\%) & No, n (\%) & & \\
\hline \multicolumn{5}{|l|}{ Gender } \\
\hline Male & $30(62.5)$ & $49(62.8)$ & 0.971 & $0.99(0.47-2.07)$ \\
\hline Female & $18(37.5)$ & $29(37.2)$ & & \\
\hline \multicolumn{5}{|l|}{ Age range (months) } \\
\hline $40.4-72$ & $19(39.6)$ & $13(16.6)$ & 0.05 & $3.28(1.43-7.51)$ \\
\hline$>73$ & $29(60.4)$ & $65(83.4)$ & & \\
\hline \multicolumn{5}{|l|}{ Asthma exacerbation } \\
\hline Present & $24(50.0)$ & $20(25.6)$ & 0.006 & $2.90(1.36-6.20)$ \\
\hline Absent & $24(50.0)$ & $58(74.4)$ & & \\
\hline \multicolumn{5}{|l|}{ Asthma severity } \\
\hline Severe persistent asthma & $27(56.2)$ & $28(35.9)$ & 0.04 & $2.30(1.03-5.12)$ \\
\hline Moderate persistent asthma & $21(43.8)$ & $50(64.1)$ & & \\
\hline \multicolumn{5}{|l|}{ Allergic rhinitis } \\
\hline Present & $41(85.4)$ & $53(67.9)$ & 0.029 & $2.76(1.09-7.02)$ \\
\hline Absent & $7(14.6)$ & $25(32.1)$ & & \\
\hline
\end{tabular}

$95 \% \mathrm{CI}=95 \%$ confidence interval; OR $=$ odds ratio. 
Table 4 - Factors associated with emergency care services in the final multivariate analysis model

\begin{tabular}{lcc}
\hline Factors & Crude OR (95\%CI) & Adjusted OR (95\%CI) \\
\hline Severe persistent asthma & $2.17(1.03-4.54)$ & $2.09(1.05-4.44)$ \\
Presence of allergic rhinitis & $2.76(1.09-7.02)$ & $2.98(1.10-8.06)$ \\
\hline
\end{tabular}

95\%CI $=95 \%$ confidence interval; OR $=$ odds ratio.

Perez Lu et al. carried out a cross-sectional outpatientbased study involving 169 children aged 2 to 14 years, who had been admitted to the emergency room due to asthma exacerbation, and found that $54 \%$ of them had AR. ${ }^{12}$

Another study, also conducted at an outpatient clinic, involved 369 patients aged 3 to 16 years who had been followed up for 1 year at an asthma referral center, and showed that $68.8 \%$ of the patients had AR. ${ }^{13}$

The follow-up of 747 children from their birth demonstrated that $42 \%$ developed concomitant asthma and AR at the age of 6 years, and that $50 \%$ of them presented with AR-compatible symptoms in the first year of life. The authors underscore the early onset of AR and its association with a higher frequency of respiratory symptoms at the age of 6 years. ${ }^{14}$

Solé et al., in a population-based study, used ISAAC phase 3 questionnaire and found remarkable differences across the cities they analyzed. The largest prevalence rates of AR among school-aged children (39.8\%) and adolescents (47.4\%) were observed in Salvador and Belém, respectively. ${ }^{15}$

Other studies show that AR coexists with asthma in 75 to $80 \%$ of asthmatic patients. ${ }^{3,4}$

The percentage of patients with AR in the present study amounted to $74.6 \%$ (95\%CI 65.9-81.7), which is in agreement with the rates described in the literature (38.9 to $80 \%$ ).

The medical literature has pointed out that the fact that the diagnosis of AR based on clinical criteria worsens asthma management ${ }^{16}$ is far more important than the association between asthma and AR. In outpatient-based studies, the prevalence of AR is higher than in population-based ones.

In the present study, all children participated in an asthma control program with regular follow-up visits and free-ofcharge dispensation of beclomethasone dipropionate. Nevertheless, $34.9 \%$ of them presented with acute asthma episodes, which therefore failed to be controlled.

The fact that none of the patients was referred to the outpatient clinic with prior diagnosis of AR, established by the basic health service, deserves some considerations. On the one hand, this may indicate the difficulty in diagnosing this comorbidity in smaller children; on the other hand, this shows the necessity of more accurate identification of AR in asthmatic patients. These results are consistent with those of a study conducted in a Peruvian emergency room, where 54.4\% of 169 children with asthma exacerbation had AR, but only $10.7 \%$ of them had been previously diagnosed with AR. ${ }^{12}$

The patients in the present study who had AR were the ones with the highest compliance rates. The mean compliance rate amounted to 64.4 vs. $47.4 \%(p=0.007)$ and, in $\mu \mathrm{g} /$ day, it amounted to $452.3 \pm 183.1$ and $311 \pm 218$ ( $p=$ 0.001 ) for those who did not have this comorbidity. Despite the higher treatment compliance, asthma could not be managed in patients with AR, who revealed a nearly threefold likelihood of necessitating emergency care due to acute asthma episodes. $^{7}$

These results concur with those of a historical cohort study by Thomas et al., using data from the dispensary system. The authors observed that children with concomitant asthma and AR had greater chances of receiving lower (OR 1.81, 95\%CI 1.05-3.14) and higher (OR 5.60,95\%CI 2.11-14.88) doses of inhaled corticosteroids than children with asthma but without AR. Notwithstanding the wider use of inhaled corticosteroids, children with AR were associated with a more frequent use of health services.

The factors associated with emergency care services found in the present study were the presence of AR and asthma severity. These findings deserve some considerations. The first one concerns the fact that patient's attention is restricted to the use of inhaled corticosteroids only. Possibly, in some of these patients, the association of a long-acting bronchodilator may have reduced the number of acute asthma episodes, since $47.3 \%$ of the patients had severe persistent asthma. These patients were prescribed doses of $750 \mu \mathrm{g}$ (500 to $1,000 \mu \mathrm{g}) /$ day. They actually needed doses of $513.5 \mu \mathrm{g} /$ day, which is regarded as the mean dose. In these cases, the literature recommends using a long-acting bronchodilator as an alternative option. ${ }^{9}$

Another aspect that should be considered is that the standardized program includes only dextrochlorpheniramine for the treatment of AR. For some patients, this approach may not have been sufficient to control AR.

The data obtained herein are consistent with those described in the literature, which indicate that the control of asthma requires the management of asthma and AR with ascending drug therapies. ${ }^{9,10}$ 
Cristal-Peters et al. conducted a historical cohort study using data from dispensaries and found out that patients treated for AR were $50 \%$ less likely to necessitate emergency care than untreated patients. The definition of AR and its treatment were based on at least two prescriptions of oral antihistamines and topical corticosteroids, as documented in the dispensary records. The authors also stated that, due to the different mechanisms of action of these two drugs, their effects may be different on asthma, but as $61 \%$ of patients received both drugs, the differences between the two drugs are unlikely to have influenced the final outcome. ${ }^{17}$

Adams et al., in a historical cohort study, also used data from dispensaries and categorized patients according to the use of topical corticosteroids and oral antihistamines in order to assess the use of these drugs and their association with the need for emergency care caused by acute asthma episodes. The likelihood of emergency care use by those treated with antihistamines was indeterminate. Those treated with topical nasal corticosteroids, having received one to three prescriptions and more than three dispensations a year, showed a lower chance of emergency care use. ${ }^{18}$

The efficacy of the unified treatment for AR and asthma, which consists of the use of a dose-metered beclomethasone aerosol, inhaled through a face mask coupled to a large valved spacer, was confirmed and may be an alternative to the treatment of AR and asthma in public health programs. ${ }^{19}$

The findings obtained here should be critically evaluated and the limitations of the study should be taken into account as well. This study was conducted at a referral outpatient clinic; therefore, its characteristics concern patients with moderate to severe asthma, but include those of patients referred by basic health services who are treated at the outpatient clinic on a regular basis. Patients with severe asthma are the ones who most often use health services, and in our patient population, the prevalence of AR might indicate a higher asthma severity.

The study design - cross-sectional in the third year of treatment - was due to the fact that some children were referred to the outpatient clinic at the age of 1 year. Given the difficulties in making the diagnosis of asthma and AR in this age group after 3 years of follow-up, the patients' medical records already indicated the diagnosis of both diseases.

On the other hand, cross-sectional studies only describe the association between events without defining the causality between them. Our study design does not allow us to infer that the poor management of asthma episodes resulted from the fact that patients were treated for AR using only dextrochlorpheniramine. To assess the impact of several AR treatments on emergency care services, clinical and placebocontrolled trials are necessary. Patients with AR needed higher doses of inhaled corticosteroids, and the presence of AR associated with asthma severity was an independent factor for the use of emergency care for the management of acute asthma.
The present study, in agreement with the guidelines established by ARIA, was based on clinical criteria for the diagnosis of $A R$. The possibility of a patient having received other drugs than beclomethasone and dextrochlorpheniramine from outside the dispensary system of the Wheezing Child Program is negligible. ${ }^{20}$ We used the dispensary system to calculate compliance rates, which is a useful method for detecting patients with partial compliance that offers a great potential for the assessment of compliance rates in the public health system. ${ }^{21,22}$

In conclusion, the findings of the present study reveal a high prevalence of AR among patients with moderate to severe persistent asthma treated at a referral outpatient clinic. The factors associated with the use of emergency care were the presence of $A R$ and asthma severity. The results point to the necessity of detecting AR as early as possible in asthmatic patients and of offering other therapies for the treatment of $A R$ and asthma in public health programs. These measures would ensure better quality of care, optimize asthma control and reduce costs.

\section{Acknowledgements}

The authors are grateful to the authorities at the Municipal Department of Health of Belo Horizonte, to the nursing staff, especially Elida Torres, Ana Cruz and Cristina Rangel from the Secondary Referral Unit Campos Sales, without whom this study would not have been possible.

\section{References}

1. Cruz AA. The 'united airways' require an holistic approach to management. Allergy. 2005;60:871-4.

2. Camargos PA, Rodrigues ME, Solé D, Scheinmann P. Asma e rinite alérgica como expressão de uma única doença: um paradigma em construção. J Pediatr (Rio J). 2002;78 Supl 2:S123-8.

3. Passalacqua G, Ciprandi G, Canonica WC. The nose-lung interaction in allergic rhinitis and asthma: united airways disease. Curr Opin Allergy Clin Immunol. 2001;1:7-13.

4. Kapsali T, Horowitz E, Miemer F, Togias A. Rhinitis is ubiquitous in allergic asthmatics. J Allergy Clin Immunol. 1997;99:S138.

5. Malone DC, Lawson KA, Smith DH, Arrighi HM, Battista C. A cost of illness study of allergic rhinitis in the United States. J Allergy Clin Immunol. 1997; 99(1 Pt 1):22-7.

6. Law AW, Reed SD, Sundy JS, Schulman KA. Direct costs of allergic rhinitis in the United States: estimates from the 1996 Medical Expenditure Panel Survey. J Allergy Clin Immunol. 2003; 111:296-300.

7. Thomas M, Kocevar VS, Zhang Q, Yin DD, Price D. Asthmarelated health care resource use among asthmatic children with and without concomitant allergic rhinitis. Pediatrics. 2005; 115:129-34.

8. Yawn PB, Yunginger JW, Wollan PC, Reed CE, Silverstein MD, Harris AG. Allergic rhinitis in Rochester, Minnesota residents with asthma: frequency and impact on health care charges. J Allergy Clin Immunol. 1999;103(1 Pt 1):54-9. 
9. National Heart, Lung, and Blood Institute, National Institutes for Health. Global strategy for asthma management and prevention. NHLBI/WHO workshop report. Bethesda: National Institute of Health; 2002.

10. Bousquet J, Van Cauwenberge P, Khaltaev N; Aria Workshop Group; World Health Organization. Allergic rhinitis and its impact on asthma ARIA workshop report. J Allergy Clin Immunol. 2001; 108(5 Suppl):S147-334.

11. Lasmar LM, Calazans GM, Fontes MJ, Camargos PA. Programa Criança que Chia, Belo Horizonte, MG. Experiência mineira com um programa municipal de atendimento à criança asmática. In: Neto AC, editor. Asma em saúde pública. São Paulo: Manole; 2007. p. 93-101.

12. Perez Lu JE, Centeno H J, Chiarella Ortigoza P, Perez Lu LE, Sialer Chavez ST. Prevalencia de rinitis alérgica en pacientes pediátricos que acuden al servicio de emergencia del Hospital Nacional Cayetano Heredia por crisis de asma. Rev Med Hered. 2003; 14:11-116.

13. Kocabas CN, Civelek E, Sackesen C, Orhan F, Tuncer A, Adalioglu G, et al. Burden of rhinitis in children with asthma. Pediatr Pulmonol. 2005;40:235-40.

14. Wright AL, Holberg CJ, Martinez FD, Halonen M, Morgan W, Taussig LM. Epidemiology of physician-diagnosed allergic rhinitis in childhood. Pediatrics. 1994;94(6 Pt 1):895-901.

15. Solé D, Wandalsen GF, Camelo Nunes IC, Naspitz CK, ISAAC Brazilian Group. Prevalence os symptoms of asthma, rhinitis, and atopic eczema among Brazilian children and adolescents identified by the International Study of Asthma and Allergies in Childhood (ISAAC) - Phase 3. J Pediatr (Rio J). 2006;82:341-6.

16. Price D, Bond C, Bouchard J, Costa R, Keenan J, Levy ML, et al. International Primary Care Respiratory Group (IPCRG) Guidelines: management of allergic rhinitis. Prim Care Respir J. 2006;15:58-70.
17. Crystal-Peters J, Neslusan C, Crown WH, Torres A. Treating allergic rhinitis in patients with comorbid asthma: the risk of asthma related hospitalizations and emergency department visits. J Allergy Clin Immunol. 2002;109:57-62.

18. Adams RJ, Fuhlbrigge AL, Finkelstein JA, Weiss ST. Intranasal steroids and risk of emergency department visits for asthma. JAllergy Clin Immunol. 2002; 109:636-42.

19. Camargos PAM, Ibiapina CC, Lasmar LMLBF, Cruz A A. Obtaining concomitant control of allergic rhinitis and asthma with a nasally inhaled corticosteroid. Allergy.2007; 62: 310-316.

20. Adams RJ, Fuhlbrigge A, Finkeslstein JA, Lozano P, Livingston $\mathrm{JM}$, Weiss $\mathrm{KB}$, et al. Impact of inhaled antiinflammatory therapy on hospitalization and emergency department visits for children with asthma. Pediatrics. 2001; 107:706-11.

21. Bender $B$, Milgrom $H$, Rand $C$. Nonadherence in asthmatic patients: is there a solution to the problem? Ann Allergy Asthma Immunol. 1997;79:177-85; quiz 185-6.

22. Bender B, Milgrom $\mathrm{H}$, Apter A. Adherence intervention research: what have we learned and what do we do next? J Allergy Clin Immunol. 2003;112:489-94.

Correspondence:

Profa. Laura Maria Belizário Lasmar

Departamento de Pediatria, Faculdade de Medicina

Universidade Federal de Minas Gerais

Av. Alfredo Balena, 190/4061

CEP 30130-100 - Belo Horizonte, MG - Brazil

Tel.: + 55 (31) 3248.9773

Fax: +55 (31) 3248.9664

E-mail: laurabl@uol.com.br 